\title{
Scarless laparoscopic repair of epigastric hernia in children
}

\author{
J. Moreira-Pinto ${ }^{1,2,3} \cdot$ J. Correia-Pinto ${ }^{1,2,3}$
}

Received: 12 November 2014/ Accepted: 13 March 2015/Published online: 20 March 2015

(C) Springer-Verlag France 2015

\begin{abstract}
Background Despite the small size of the incision, the scar left by open repair of epigastric hernia in children is unaesthetic. Few laparoscopic approaches to epigastric hernia repair have been previously proposed, but none has gain wide acceptance from pediatric surgeons. In this study, we present our experience with a scarless laparoscopic approach using a percutaneous suturing technique for epigastric hernia repair in children.

Methods Ten consecutive patients presenting with epigastric hernia $15 \mathrm{~mm}$ or further from the umbilicus were submitted to laparoscopic hernia repair. A 5-mm $30^{\circ}$-angle laparoscope is introduced through a umbilical trocar and a 3-mm laparoscopic dissector is introduced through a stab incision in the right flank. After opening and dissecting the parietal peritoneum, the fascial defect is identified and closed using $2-0$ polyglactin thread through a percutaneous suturing technique. Intraoperative and postoperative clinical data were collected.

Results All patients were successfully submitted to laparoscopic epigastric hernia repair. Median age at surgery
\end{abstract}

Electronic supplementary material The online version of this article (doi:10.1007/s10029-015-1369-2) contains supplementary material, which is available to authorized users.

J. Moreira-Pinto

moreirapinto@gmail.com

1 Life and Health Sciences Research Institute (ICVS), School of Health Sciences, University of Minho, Campus de Gualtar, 4710-057 Braga, Portugal

2 ICVS/3B's-PT Government Associate Laboratory, Braga/ Guimarães, Portugal

3 Department of Pediatric Surgery, Hospital de Braga, Braga, Portugal was 79 months old and the median distance from the umbilicus to the epigastric defect was $4 \mathrm{~cm}$. Operative time ranged from 35 to $75 \mathrm{~min}$. Every hernia was successfully closed without any incidents. Follow-up period ranges from 2 to 12 months. No postoperative complications or recurrence was registered. No scar was visible in these patients.

Conclusion This scarless laparoscopic technique for epigastric hernia repair is safe and reliable. We believe this technique might become gold standard of care in the near future.

Keywords Epigastric hernia · Children · Laparoscopy · Surgical repair - Percutaneous suturing · Umbilicus

\section{Introduction}

Epigastric hernia is a ventral hernia presenting as a fascial defect along the linea alba anywhere from the xyphoid process to the umbilicus. It represents $4 \%$ of all pediatric hernias presenting in children. Most surgeons recommend surgical repair at time of diagnosis, especially if it is symptomatic [1]. Traditionally, surgical repair has been performed through a $1-2 \mathrm{~cm}$ transverse incision over the defect. Identification of the fascial defect can sometimes be difficult, which can result in a bigger incision. Despite the small size of the incision, the scar left by open repair of epigastric hernia is unaesthetic. Few laparoscopic approaches to epigastric hernia repair have been previously proposed, but none has gain wide acceptance from pediatric surgeons. In this study, we present our experience with a scarless laparoscopic approach using a percutaneous suturing technique for epigastric hernia repair. 


\section{Methods}

From August 2013 to June 2014, eight consecutive patients presenting with epigastric hernia located $15 \mathrm{~mm}$ or further from the umbilicus were enrolled in this study. A novel laparoscopic technique for hernia repair was performed in an outpatient setting. All patients were anesthetized and ventilated using an endotracheal tube. Intraoperative and postoperative clinical data were collected.

\section{Surgical technique}

The exact site of the epigastric hernia is marked preoperatively. The patient is placed in a supine position. The surgeon stands on the patient's left side and the assistant stands in the patient's right side. The monitor is placed following patient's head.

An intra-umbilical incision is made and a $5 \mathrm{~mm}$ trocar is inserted. An $8-10 \mathrm{mmHg}$ pneumoperitoneum is maintained using carbon dioxide insufflation. A 5-mm $30^{\circ}$-angle laparoscope (Hopkins II telescope, 26011BA, Karl Storz, Tuttligen, Germany) is introduced and the abdominal wall is inspected.

Pressing the abdominal wall over the previously marked hernia helps identifying the fascial defect. A 3-mm laparoscopic dissector (30351, Karl Storz) is introduced through a stab incision in the left flank as shown in Fig. 1. The peritoneum is incised and the fascial defect is exposed, using electrocoagulation as needed. The assistant may press the abdominal wall just above the defect to better expose the fascial defect (Fig. 2). Any incarcerated fat is pulled back into the abdominal cavity (Fig. 3, Video 1).

Afterwards, a 2-0 polypropylene suture is threaded through a $16 \mathrm{G}$ needle and inserted through one of the sides of the defect in order to create a loop inside the abdomen (Fig. 4, Video 2). The same $16 \mathrm{G}$ needle is used to pass a

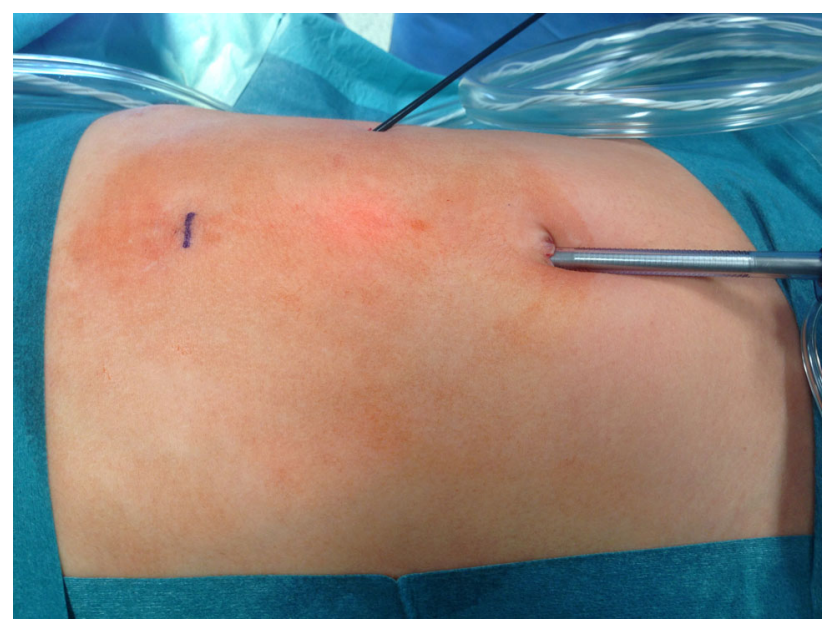

Fig. 1 An intra-umbilical incision is made and a $5 \mathrm{~mm}$ trocar is inserted. A 5-mm $30^{\circ}$-angle laparoscope is used and 3-mm laparoscopic dissector is introduced through a stab incision in the left flank

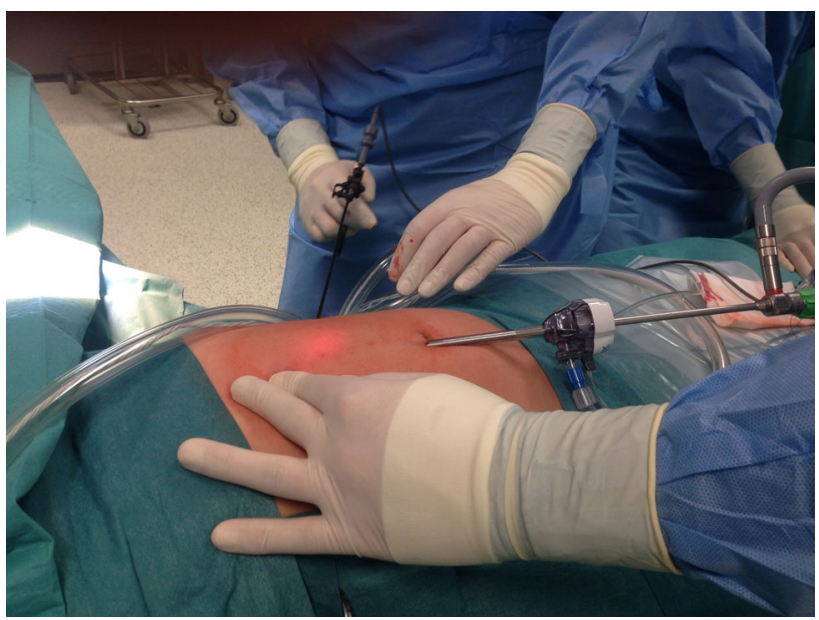

Fig. 2 The assistant may press the abdominal wall just above the defect to better expose the fascial defect

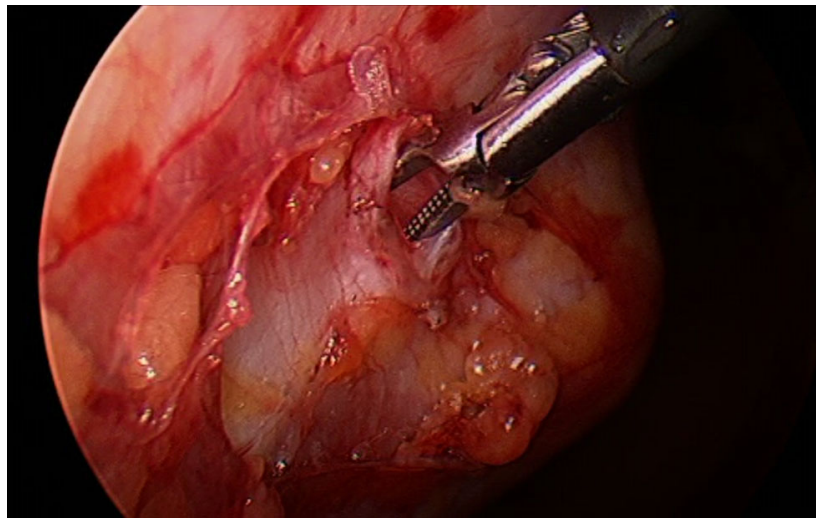

Fig. 3 The fascial defect is identified and any incarcerated fat is pull out of the epigastric hernia

different 2-0 polyglactin suture going through the other side of the defect and into the loop. Pulling the loop back out results in a 2-0 polyglactin stitch approximating both sides of the fascial defect. A knot is tied extracorporeally. It is important to use the exact same site every time one enters the skin so that the knot can enter deeply into the subcutaneous tissue. Consecutive stitches are passed using the same technique depending on the size of the defect.

The umbilical port is closed using 2-0 polyglactin suture and skin is closed using 5-0 poliglecaprone suture. Stab incision and the needle site of entry are protected using steri-strips (Fig. 5).

\section{Results}

Ten patients (5 male and 5 female) were submitted to scarless laparoscopic repair of their epigastric hernia. Age at surgery ranged from 23 to 142 months old 

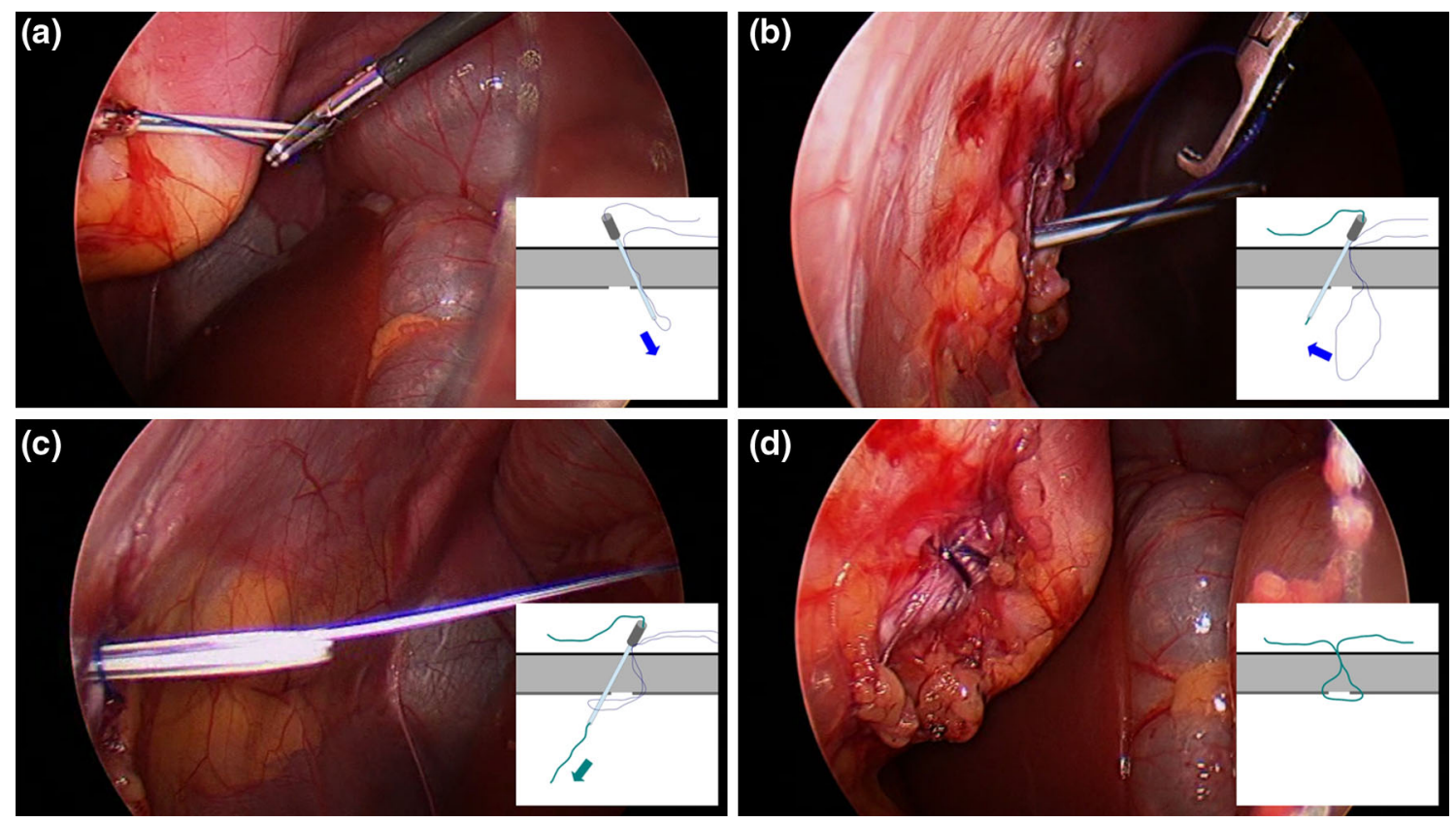

Fig. 4 Arrows represent grasping action of the laparoscopic dissector. a A 2-0 polypropylene suture is threaded through a $16 \mathrm{G}$ needle and inserted through one of the sides of the defect. $\mathbf{b}$ A loop is created inside the abdomen with the help of the dissector. c The same $16 \mathrm{G}$

needle is used to pass a different $2-0$ polyglactin suture going through the other side of the defect and into the loop. $\mathbf{d}$ Pulling the loop back out results in a $2-0$ polyglactin stitch approximating both sides of the fascial defect. A knot is tied extracorporeally

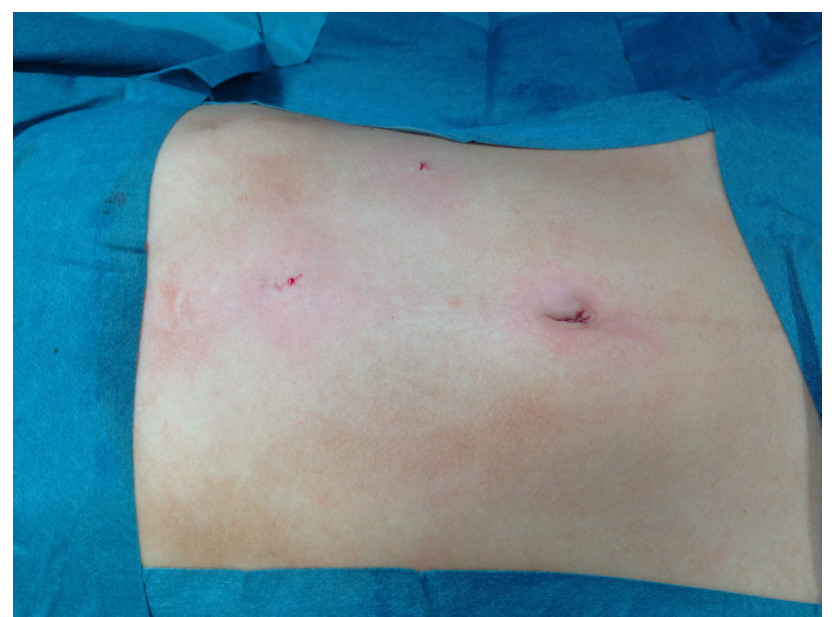

Fig. 5 The umbilical port is closed using 2-0 polyglactin suture and skin is closed using 5-0 poliglecaprone suture. Stab incision and the needle site of entry are protected using steri-strips

(median $=79$ months old). Operative time ranged from 35 to $75 \mathrm{~min}$ (median $=50 \mathrm{~min}$ ). Median distance from the umbilicus to the epigastric defect was $4 \mathrm{~cm}$ (ranged from 2 to $9 \mathrm{~cm}$ ). All procedures were completed without incidents. In one case, identifying the defect obliged a partial section of the falciform ligament of the liver. One other patient had a left-sided paraumbilical melanocytic nevus excised simultaneously and we used the site of excision to insert the

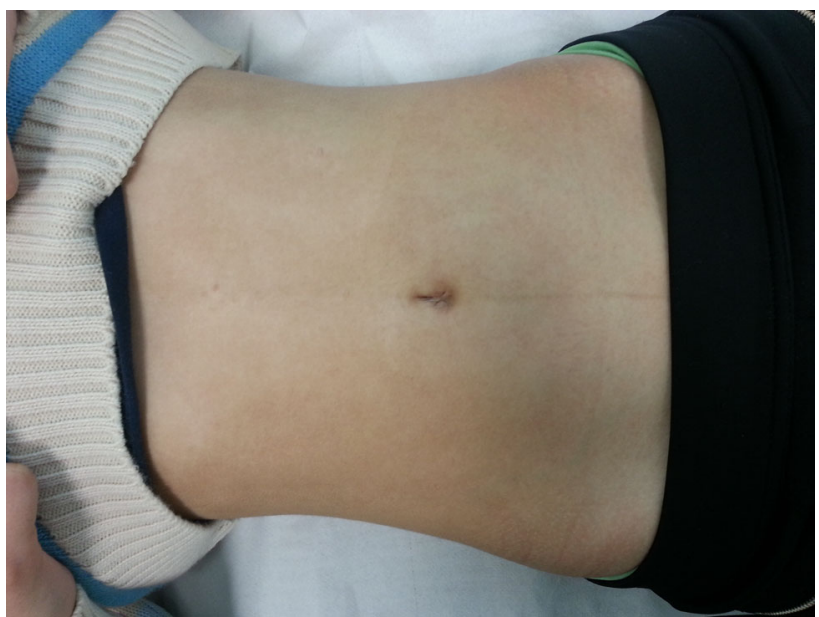

Fig. 6 Both umbilical and stab incision scars were barely visible by 1 month postoperatively (same patient as in Fig. 5)

3-mm dissector. In patient number 7, 3 fascial defects were identified and repaired in the same surgery.

Follow-up period ranges from 2 to 12 months (median $=8$ months). Every hernia was successfully closed without any recurrence. No postoperative complication was registered. Cosmetic outcome was favorable in all patients as both umbilical and stab incision scars were barely visible by 1 month postoperatively (Fig. 6). Parent's satisfaction was subjectively excellent. 


\section{Discussion}

Although a common procedure in pediatric surgeon's practice, there is not much written in the scientific literature on epigastric hernia. According to Coats et al. [1] most are asymptomatic $(63 \%)$ and reducible $(65 \%)$. Repair of an epigastric hernia classically involves a transverse incision over the site of the defect, removal of incarcerated fat and suture closure of the linea alba. Skin incision relates to a higher risk of complications such as infection, hypertrophic scarring, dehiscence, and infection. Even when no complication exists, having an $1-2 \mathrm{~cm}$ scar is a high price to pay for such a benign condition. Moreover, some patients have multiple fascial defects $(13 \%)$ requiring multiple scars [1].

In the past, Albanese et al. [2] proposed a laparoscopic technique to epigastric hernia repair. The authors suggested inserting two 3-mm trocars through the lateral abdominal and lower chest wall and performing a complex onehanded intracorporeal suture assisted with a laparoscopic knot pusher. Because it was in fact difficult to perform, sometimes a third port was needed. We believe that this fact was enough to justify pediatric surgeons resistance in using this technique.

In December 2012, Tatekawa et al. [3] published a case report where they performed a single-port laparoscopic epigastric hernia repair. The authors inserted a $5 \mathrm{~mm}$ trocar in umbilicus through which they introduced a laparoscope. They inspected the abdominal wall and found out that the defect was not able to be detected. They used subcutaneous saline injection at the site of the defect which made the peritoneum protrude inward. Without exposing the fascial defect they performed five stitches using a $20 \mathrm{G}$ epidural needle. The technique for suture was the same as described above, which is inspired in the percutaneous Internal ring suture (PIRS) described by Patkowski et al. [4]. Although no conclusion can be taken by a single successful repair, we believe that the absence of a clear visualization of the fascial defect might favor failure of the hernia repair.

In the 2013 International Pediatric Endoscopic Group Annual Meeting, Babsail et al. [5] presented single-incision pediatric endosurgical epigastric hernia repair. The authors introduced two trocars (5- and 3-mm) through a single umbilical incision. They opened the peritoneum and dissected the fascial defect. The hernia repair was performed using looped 4-0 polypropylene sutures introduced through a 17 gauge spinal needle using the same percutaneous technique as previously described. During the video presentation, it was clear that clinching of the instruments was a problem, especially for surgeons not familiar with single-port techniques.

In our department, epigastric hernias located closer than $15 \mathrm{~mm}$ from the umbilicus are repaired using an umbilical incision. Dissecting the linea alba upwards makes it easy to identify and repair hernias presenting just above the umbilicus. For epigastric hernias located $15 \mathrm{~mm}$ or further from the umbilicus we started using the scarless laparoscopic approach. Our laparoscopic technique respects the traditional and long experienced open epigastric hernia repair technique. Using a 3-mm laparoscopic dissector inserted through a small stab incision, we were able to dissect the peritoneum and clearly expose the fascial defect without clinching of the instruments. Moreover, having a dissector inside the abdomen permits pulling the incarcerated fat into the abdominal cavity and facilitates the percutaneous suture technique, because one can manipulate the loop easier and pull the absorbable suture through it. Because we do not insert a trocar for the 3-mm dissector to go in, the scar is barely visible after 1 month postoperatively. As for the umbilicus, for the insertion of a $5-\mathrm{mm}$ trocar through it leaves no scar. In fact, the umbilicus is seen as human's natural embryonic scar. This is why the laparoscopic technique that we propose has such an excellent cosmetic outcome.

Although we have a small case in our series, the lack of incidents intraoperatively, the absence of postoperative complications and zero recurrences makes us believe that this technique is safe and reliable. Scarless laparoscopic repair for epigastric hernia is a quick and simple technique that respects the open surgery repair principles, and it just requires a $5 \mathrm{~mm}$ trocar, a $30^{\circ}$-angle laparoscope and a 3-mm dissector. We believe this technique might become gold standard of care in the near future.

Conflict of interest JCP is a consultant for Karl Storz. JMP has no conflict of interests to declare.

\section{References}

1. Coats RD, Helikson MA, Burd RS (2000) Presentation and management of epigastric hernias in children. J Pediatr Surg 35:1754-1756

2. Albanese CT, Rengal S, Bermudez D (2006) A novel laparoscopic technique for the repair of pediatric umbilical and epigastric hernias. J Pediatr Surg 41:859-862

3. Tatekawa Yukihiro, Yamanaka Hiroaki, Hasegawa Toshimichi (2013) Single-port laparoscopic repair of an epigastric hernia using an epidural needle. Int J Surg Case Rep 4:262-264

4. Patkowski D, Czernik J, Chrzan R, Jaworski W, Apoznański W (2006) Percutaneous internal ring suturing: a simple minimally invasive technique for inguinal hernia repair in children. $\mathrm{J} \mathrm{La}-$ paroendosc Adv Surg Tech A 16:513-517

5. Babsail A, Abelson JS, Liska D, Muensterer OJ (2014) Singleincision pediatric endosurgical epigastric hernia repair. Hernia 18:357-360 